\title{
The effect of hypnosis on pain and peripheral blood flow in sickle-cell disease: a pilot study
}

\author{
Ravi R Bhatt' \\ Sarah R Martin' \\ Subhadra Evans ${ }^{2}$ \\ Kirsten Lung' \\ Thomas D Coates 3,4 \\ Lonnie K Zeltzer ${ }^{1}$ \\ Jennie C Tsao' \\ 'UCLA Pediatric Pain and Palliative \\ Care Program, Division of \\ Hematology-Oncology, Department \\ of Pediatrics, David Geffen School \\ of Medicine at UCLA, Los Angeles, \\ CA, USA; ${ }^{2}$ School of Psychology, \\ Deakin University, Geelong, VIC, \\ Australia; ${ }^{3}$ Department of Pediatrics, \\ Keck School of Medicine, University \\ of Southern California, ${ }^{4}$ Children's \\ Center for Cancer and Blood \\ Diseases, Children's Hospital Los \\ Angeles, Los Angeles, CA, USA
}

This article was published in the following Dove Press journal: Journal of Pain Research

14 July 2017

Number of times this article has been viewed

Background: Vaso-occlusive pain crises (VOCs) are the "hallmark" of sickle-cell disease (SCD) and can lead to sympathetic nervous system dysfunction. Increased sympathetic nervous system activation during VOCs and/or pain can result in vasoconstriction, which may increase the risk for subsequent VOCs and pain. Hypnosis is a neuromodulatory intervention that may attenuate vascular and pain responsiveness. Due to the lack of laboratory-controlled pain studies in patients with SCD and healthy controls, the specific effects of hypnosis on acute pain-associated vascular responses are unknown. The current study assessed the effects of hypnosis on peripheral blood flow, pain threshold, tolerance, and intensity in adults with and without SCD.

Subjects and methods: Fourteen patients with SCD and 14 healthy controls were included. Participants underwent three laboratory pain tasks before and during a 30-minute hypnosis session. Peripheral blood flow, pain threshold, tolerance, and intensity before and during hypnosis were examined.

Results: A single 30-minute hypnosis session decreased pain intensity by a moderate amount in patients with SCD. Pain threshold and tolerance increased following hypnosis in the control group, but not in patients with SCD. Patients with SCD exhibited lower baseline peripheral blood flow and a greater increase in blood flow following hypnosis than controls.

Conclusion: Given that peripheral vasoconstriction plays a role in the development of VOC, current findings provide support for further laboratory and clinical investigations of the effects of cognitive-behavioral neuromodulatory interventions on pain responses and peripheral vascular flow in patients with SCD. Current results suggest that hypnosis may increase peripheral vasodilation during both the anticipation and experience of pain in patients with SCD. These findings indicate a need for further examination of the effects of hypnosis on pain and vascular responses utilizing a randomized controlled trial design. Further evidence may help determine unique effects of hypnosis and potential benefits of integrating cognitive-behavioral neuromodulatory interventions into SCD treatment.

Keywords: sickle-cell disease, pain, hypnosis, blood

\section{Introduction}

Sickle-cell disease (SCD) affects up to 100,000 Americans at a rate of one of every 500 African-American births. ${ }^{1}$ Vaso-occlusive pain crises (VOCs) are considered the "hallmark" of SCD. VOCs occur frequently and may lead to development of chronic pain, such that $30 \%$ of sampled patients with SCD have reported pain nearly every day. ${ }^{1}$ It has been proposed that continuous allostatic stress load (eg, recurring VOCs) can initiate a cascade of physiological changes in patients who experience recurrent pain. $^{2}$ Enhanced sympathetic nervous system activation, parasympathetic nervous

Correspondence: Ravi R Bhatt UCLA Pediatric Pain and Palliative Care Program, Department of Pediatrics, David Geffen School of Medicine at UCLA, 10833 Le Conte Ave - MDCC 22-464, Los Angeles, CA 90095, USA Email ravibhatt@mednet.ucla.edu 
system withdrawal, and subsequent peripheral vaso-occlusion in individuals with $\mathrm{SCD}^{3}$ result in hypoxic tissue damage, which if recurrent may ultimately lead to changes to the peripheral and central nervous systems. Indeed, transgenic sickle mice exhibit hyperalgesia and neuronal hypersensitivity and individuals with SCD show hypersensitivity (lower pain thresholds) to thermal stimuli, ${ }^{4}$ providing support for the hypothesis that changes in peripheral and central neuronal sensory signaling may lead to changes in pain responsiveness. ${ }^{5,6}$ If this hypothesis is validated, SCD patients with frequent $^{7}$ VOCs may be at higher risk for the development of altered pain-response mechanisms and thus at higher risk for the development of chronic pain. Therefore, examination of interventions that may address factors associated with the initiation of VOCs (eg, stress, pain, peripheral blood flow) is needed.

Neuromodulatory interventions target cortical pain modulation and have been shown to have a favorable effect on central and peripheral physiological processes related to the experience of acute and chronic pain; ${ }^{8,9}$ however, limited data exist on the effect of neuromodulatory interventions on mechanisms underlying VOCs (ie, microvascular stress/pain responses) in patients with SCD. Engagement in hypnosis, a cognitive-based neuromodulatory intervention, has been associated with decreases in acute and chronic pain. ${ }^{8}$ Specifically, engagement in hypnosis is associated with changes in central neuromodulatory and autonomic processes. ${ }^{9-13}$

Despite limited studies in SCD samples, preliminary evidence suggests that hypnosis may be a promising intervention to mitigate pain and the acute-stress response. One study described patients with SCD reporting less pain overall as well as during VOCs following a hypnosis intervention. ${ }^{14}$ A case study of a longitudinal hypnosis intervention in two adolescents with SCD reported that the adolescents had a feeling of overall warmth and a flushed appearance, presumed to be associated with peripheral vasodilation. ${ }^{15}$ Other studies reported preliminary evidence of hypnosis-induced vasodilatation in healthy controls and other pain conditions. ${ }^{14,15}$ These results suggest that hypnosis may be a beneficial cognitivebehavioral intervention for individuals with SCD. While prior studies have shown promise for hypnosis as a clinical tool for pain control in SCD, the mechanisms underlying the effects of hypnosis on acute pain in this population remain unknown. Because the clinical setting provides significant variability in pain experiences, context, and responses, a study of mechanisms of the effects of hypnosis on acute pain in patients with SCD is best studied in the controlled setting of a psychophysiological pain laboratory. Inclusion of a comparison non-SCD control group can further provide evidence on responses that may be unique to patients with SCD. Therefore, the objective of the current study was to assess the effects of hypnosis on acute experimental pain responses and the corresponding responses in microvasculature in adults with and without SCD.

\section{Specific aims and hypotheses}

The first aim of this pilot study was to assess whether baseline pain responses (ie, pain threshold, tolerance, intensity) and peripheral blood flow differed across patients with SCD and race-matched healthy controls. The second aim was to examine the effect of a brief hypnotic intervention on acute experimental pain responsiveness during a thermal pain task, as assessed by pain threshold, tolerance, and intensity. The third aim was to determine the effects of hypnosis on changes in peripheral blood flow during anticipation of the pain task and during the pain task itself. Finally, the fourth aim was to determine if the effect of hypnosis on pain responses and blood flow differed across groups.

We hypothesized that patients with SCD would have lower peripheral blood flow at baseline and demonstrate lower pain thresholds and tolerance and higher pain intensity. We hypothesized that in both groups, pain threshold and tolerance would increase and pain intensity would decrease after hypnosis. We also hypothesized that peripheral blood-flow amplitude would increase in both groups during the anticipation and experience of the pain tasks following the hypnosis intervention. Finally, due to already present vasoconstriction and reduced blood flow, we hypothesized that patients with SCD would experience more benefit from hypnosis and thus exhibit a greater change in blood flow following hypnosis. In addition, we expected that patients with SCD would demonstrate a greater increase in pain threshold and tolerance and decrease in pain intensity following hypnosis compared to healthy controls.

\section{Subjects and methods}

All procedures and methods of the current study were approved by the Medical Institutional Review Board of the University of California - Los Angeles. This trial is registered with ClinicalTrials.gov (NCT02620488).

\section{Participants}

Participants with SCD ( $\mathrm{n}=14$, eleven females, mean age 34 years, SD 12.88) and without SCD ( $n=14$, eleven females, mean age 37.23 years, SD 17.34) were recruited from various locations in the greater Los Angeles area, including the 
Cayenne Wellness Center and Children's Foundation, and various community SCD meetings in Los Angeles. Inclusion criteria for the participants with SCD were a confirmed diagnosis of SS hemoglobin, SC hemoglobin, or S-thalassemia hemoglobin. Inclusion criteria for the control population were healthy individuals (ie, no chronic illness diagnosis) whose race matched that of the SC patients. Exclusion criteria included any neurologic disorder that affected sensation, skin abnormalities/abrasions over the site of stimulus application, and any acute or chronic illness that may have impaired safety or lab performance. All participants were screened via telephone to determine eligibility. We know of only one study looking at changes in peripheral blood flow in disease populations, ${ }^{16}$ and various studies investigating peripheral blood flow following hypnotic suggestions. ${ }^{14,17}$

We chose our sample size to assess feasibility of the current protocol and to see if there was any effect on a clinically meaningful outcome that has been specifically implicated in the genesis of VOCs, and our sample comparable to past studies looking at hypnosis and peripheral blood flow.

\section{Procedure}

All procedures were approved by the Institutional Review Board of the University of California, Los Angeles and were conducted at the Pediatric Pain and Palliative Care Program Laboratory. The experimenter and the participant were seated in the same room. The participant first provided written consent and completed questionnaires. Next, a pulse-oximetry transducer was placed on the participant's left thumb to assess peripheral blood flow. The participant sat quietly for $3 \mathrm{~min}$ utes, during which baseline peripheral blood-flow data were collected. Following the baseline period, the experimenter verbally announced, "In a minute, pain task 1 will begin", which marked the start of the pain-anticipation period that lasted approximately 1 minute. The first set of pain tasks (referred to pre-hypnosis tasks) were then conducted and pain threshold and tolerance assessed. This was followed by a 3-minute recovery, or washout period, and then followed by assessing pain intensity. After the three pain tasks had been completed, a psychologist trained in medical hypnosis was then brought into the room to engage the participant in a 30-minute hypnosis session. After the psychologist had delivered hypnotic suggestions for analgesia (referred to as post-hypnosis), the three pain tasks were readministered in the same order as before hypnosis (with a 3-minute washout period following assessment of pain threshold). During the second pain task, the participant was receiving booster suggestions from the psychologist. The anticipation period before the application of the pain stimulus was investigated because it is known that the anticipation of pain has influence over activity in the somatosensory cortex, and thus can influence the pain experience itself and corresponding physiological responses. ${ }^{18}$ A summary of the study phases is depicted in Figure 1. At the end of the laboratory session, the psychologist conducted a brief exit interview and confirmed that the participant was fully alert and able to function cognitively before leaving the premises. Compensation was then provided.

\section{Measures}

\section{Peripheral blood flow}

Peripheral blood flow and the heat signal from the neurosensory analyzer were collected continuously throughout the study using the BioPac MP150 system and AcqKnowledge software (version 3.9.0) so that the timing of all of the measurements was synchronized. Continuous readings for $\mathrm{SpO}_{2}$, pulse rate, and pulse waveform were monitored using the pulse-oximetry transducer placed on the left thumb at $1,000 \mathrm{~Hz}$. The average blood-flow amplitude - defined by the distance from trough to peak on photoplethysmography - during each study phase was used for analyses. Blood-flow data were then normalized so that parametric statistical tests could be performed, and thus no unit is reported, as they were normalized.

\section{Laboratory pain responsiveness}

All nociceptive stimulation was applied using a TSA-II neurosensory analyzer (Medoc Advanced Medical Systems,

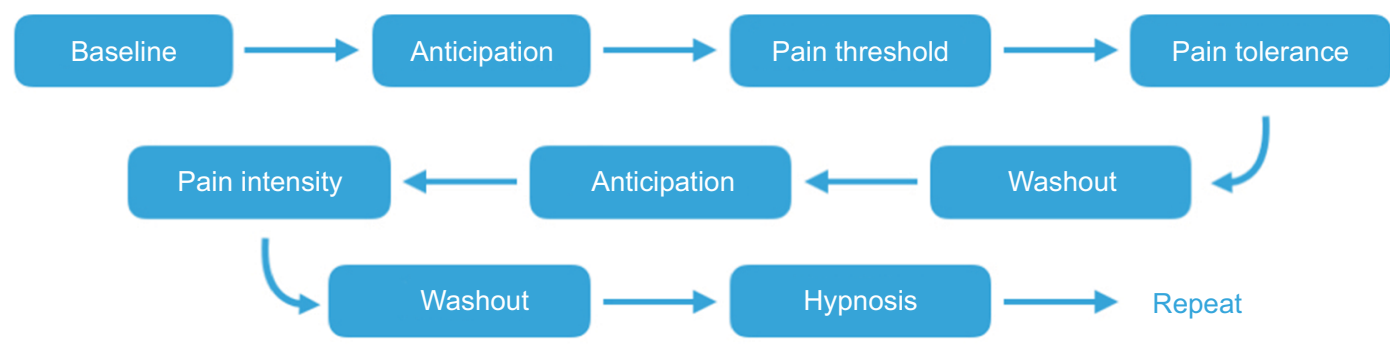

Figure I Summary of study phases before and after hypnosis. 
Ramat Yishai, Israel). The TSA-II consists of a "thermode" $(30 \times 30 \mathrm{~mm})$ starting at a temperature of $32^{\circ} \mathrm{C}$ and reaching a maximum temperature of $52^{\circ} \mathrm{C}$ to prevent tissue damage. The thermode was placed on the participant's right forearm (brachioradialis muscle) just below the joint cavity and was moved down $25.4 \mathrm{~mm}$ after each ramp was delivered. Each participant was administered a set of pain tasks twice: before and after hypnotic analgesia suggestions. Each set of pain tasks consisted of three pain tasks (ie, assessment of pain threshold, pain tolerance, and pain intensity; see Figure 1 for illustration of study procedure). This procedure was established in a previous study. ${ }^{19}$

\section{Pain threshold and tolerance}

The pain-threshold and -tolerance tasks consisted of six heat ramps. Once the ramp started, temperature rose $1^{\circ} \mathrm{C}$ per second. During the first three ramps, participants indicated when they first perceived the stimulus to be painful, which was considered their pain threshold. During the last three ramps, participants indicated when they could no longer tolerate the pain (ie, pain tolerance). Then, the threshold and tolerance ratings were averaged across all six trials (three threshold and three tolerance trials), and this value was used as the target temperature administered during the third task.

\section{Pain intensity}

The third task consisted of two heat ramps delivered at the aforementioned target temperature. Once the thermode reached this temperature, the participant was asked to rate his/her pain intensity on a $0-100$ numeric rating scale (ie, $0=$ no pain, $100=$ worst pain possible). This value was classified as pain intensity.

\section{Hypnosis intervention}

After administration of the first set of pain tasks, the psychologist greeted the patient. The participant was informed about the facts and myths about medical hypnosis and was provided an opportunity to ask questions. The hypnosis procedure was divided into five phases: 1) relaxation induction to induce a narrowed focus of attention, 2) intensification of the focused attention and involvement in imagery using a "favorite place" suggestion, 3) pre-pain-task analgesic suggestions for personally derived analgesic imagery, 4) preand within-task blood-flow suggestions targeting improved peripheral vasodilatation through imagery related to warming and water flow, and 5) posttask posthypnotic suggestions for continued comfort with an alert mind.

\section{Relaxation induction}

After the introduction, the participant was encouraged to sit in a relaxed, comfortable position with eyes closed, if so desired. The participant was then invited to experience relaxation imagery, involving relaxation throughout the body with such suggestions as "Allowing all the muscles in the shoulders to let go, relaxing, feeling the support of the chair, sinking into the chair... letting all the tension drain out of the shoulders".

\section{Intensification}

A deepening elevator exercise was then used, where the participant was instructed to imagine going down in an elevator and relaxing more deeply with each floor. This was followed by instructions to imagine a favorite place, a place that evokes feelings of relaxation and ease. The participant was invited to experience the sights, smells, and textures of this favorite place, eg, “... You inhale and smell all the delicious smells of your favorite place. Perhaps it's a beach, or a mountaintop. Somewhere you've felt warm and safe and serene and content before. You reach down and feel the ground. Is it sand slipping through the fingers, or the coolness of new-grown grass? Let's stay here, in this place, for a few moments, while you soak in the sensations."

\section{Hypnotic analgesia - suggestion I}

Next, consistent with the study by Jensen, ${ }^{20}$ analgesia suggestions were offered in which the participant was invited to imagine his or her own personal pain analgesia. Suggestions were offered for this personal analgesia to be experienced as a favorite color, a cooling balm, a pill, or any other way the subject desired. Feelings of relief and comfort were encouraged immediately following use of the analgesia.

\section{Hypnotic analgesia and vasodilatory imagery - suggestion 2}

The pain tasks were then administered a second time, while the hypnotherapist continued to support the participant in the experience of relief and comfort, eg, “... perhaps imagining feelings of relief throughout the arm, the arm feeling more and more comfortable as the medicine spreads". During the pain task, the psychologist continued to provide booster support to enhance the effects hypnotic analgesia further, eg, “... Now imagine the area of the arm experiencing heat, imagine it being completely surrounded... or completely filled... with a sensation of relief... a pleasant sensation of comfort ... you might like to picture feelings of relief spreading down the arm. Noticing how naturally, how easily, you are able to make the arm feel different and much more pleasant... even decreasing sensations from that area; as if it were disappearing". 


\section{Posthypnotic period}

After the pain tasks had been completed, the psychologist invited the participant to experience warm-bath imagery, designed to evoke vasodilatory sensations for a 5- to 7-minute posttask recovery period. Instructions included: "This bath, just the right kind of warmth. The water soothing muscles, opening the body, to relax. Such a calm, pleasant feeling. The warm water like liquid therapy, melting away troubles, washing away cares, allowing the body to relax into an even deeper state of well-being". The participant was then provided with sufficient time to return to their normal state. A countdown from 10 to 1 was used, with 1 being a state of full awareness and alertness. The full script for the hypnosis procedure is included in the Supplementary Material.

\section{Data analysis}

Shapiro-Wilk tests of normality were conducted on the primary-outcome variables (blood flow, pain threshold, pain tolerance, pain intensity) to determine appropriate statistical tests. Natural log transformations were conducted to normalize abnormal pain-responsiveness data, but the data were resistant to transformation. As such, appropriate parametric (ie, paired- or independent-sample $t$-tests) and nonparametric (ie, Wilcoxon signed-rank and Mann-Whitney $U$-tests) meandifference tests were used for analyses that included normal and abnormal variables, respectively. Independent-sample $t$-tests were utilized to determine if baseline levels differed across groups. Paired-sample mean-difference tests were conducted to determine if there was a significant change in laboratory pain responses and blood-flow amplitude following hypnosis. To compare the effect of hypnosis on blood-flow amplitude across groups, the change in amplitude following hypnosis was determined by subtracting the mean amplitude during hypnosis from the amplitude value during each posthypnosis task. Independent-sample $t$-tests were then used to compare change in blood-flow amplitude across groups. All significance testing was conducted at $\alpha=0.05$. Parametric testing used Cohen's $d$ as the effect size ( small $=0.2$, medium $=$ 0.5 , large $=0.8) .{ }^{19}$ Nonparametric testing used Pearson's $r$ as the effect size $($ small $=0.1$, medium $=0.3$, large $=0.5) .{ }^{20}$ All analyses were conducted in RStudio (version 3.2.1).

\section{Results}

\section{Preliminary results}

The sample consisted of 14 total patients with SCD (eleven females, mean age 34 years, SD 12.88) and 14 healthy controls (eleven females, mean age 37.23 years, SD 17.34). Age was not associated with baseline pain threshold $(r=-0.16, p=0.58$; $r=-0.48, p=0.09)$ or pain tolerance $(r=-0.47, p=0.09 ; r=0.11$, $p=0.7)$ in the SCD and control groups, respectively. Age was associated with baseline peripheral blood flow in patients with $\operatorname{SCD}(r=0.69, p=0.019)$, but not in controls $(r=0.32, p=0.31)$.

\section{Aim I: baseline pain threshold, tolerance, self-reported pain intensity, and blood flow}

An independent-sample $t$-test revealed that there was no difference in baseline pain threshold $\left(t_{25.96}=-0.07, d=0.03\right.$; $p=0.94)$, tolerance $\left(t_{25.82}=-0.25, d=0.09 ; p=0.81\right)$, or intensity $\left(t_{25.38}=-0.75, d=0.29 ; p=0.457\right)$. The same test revealed controls had higher peripheral blood flow during baseline than patients with $\operatorname{SCD}\left(t_{21.17}=2.54, d=1.01 ; p=0.019\right)$, but there were no group differences during hypnosis $\left(t_{19.14}=1.61\right.$, $d=0.672 ; p=0.12$ ). No other differences between groups were found across tasks in blood flow. Means of each task across groups are reported in Table 1.

\section{Aim 2: effects of hypnosis on pain threshold, tolerance, and self-reported pain intensity}

Paired $t$-tests and Wilcoxon signed-rank tests were conducted within groups to determine the effect of hypnosis on controls and patients with SCD for each task. Results revealed that

Table I Laboratory pain sensitivity and peripheral blood flow during each phase of the study

\begin{tabular}{|c|c|c|c|c|}
\hline \multirow[t]{2}{*}{ Pain } & \multicolumn{2}{|c|}{ Controls $(n=14)$} & \multicolumn{2}{|c|}{$\operatorname{SCD}(n=14)$} \\
\hline & $\begin{array}{l}\text { Mean } \\
\left({ }^{\circ} \mathrm{C}\right)\end{array}$ & SD & $\begin{array}{l}\text { Mean } \\
\left({ }^{\circ} \mathrm{C}\right)\end{array}$ & SD \\
\hline Pre-hypnosis pain threshold & 43.56 & 3.95 & 43.86 & 3.93 \\
\hline Pre-hypnosis pain tolerance & 47.12 & 2.83 & 47.39 & 2.57 \\
\hline Pre-hypnosis pain intensity & 31.68 & 22.44 & 38.64 & 26.27 \\
\hline Post-hypnosis pain threshold & 45.9 & 3.81 & 44.45 & 3.71 \\
\hline Post-hypnosis pain tolerance & 48.48 & 1.98 & 47.7 & 3 \\
\hline Post-hypnosis pain intensity & 18.57 & 18.26 & 33.46 & 28.23 \\
\hline \multirow[t]{2}{*}{ Blood flow } & \multicolumn{2}{|c|}{ Controls $(n=13)$} & \multicolumn{2}{|c|}{$\operatorname{SCD}(n=I I)$} \\
\hline & Mean & SD & Mean & SD \\
\hline Baseline** & 0.82 & 0.07 & 0.76 & 0.05 \\
\hline Pre-hypnosis anticipation I & 0.81 & 0.08 & 0.76 & 0.05 \\
\hline Pre-hypnosis pain task I & 0.82 & 0.05 & 0.77 & 0.05 \\
\hline Pre-hypnosis anticipation 2 & 0.81 & 0.09 & 0.76 & 0.08 \\
\hline Pre-hypnosis pain task $2^{*}$ & 0.81 & 0.06 & 0.75 & 0.08 \\
\hline Hypnosis & 0.8 & 0.05 & 0.76 & 0.06 \\
\hline Post-hypnosis anticipation I* & 0.79 & 0.06 & 0.82 & 0.05 \\
\hline Post-hypnosis pain task I & 0.79 & 0.05 & 0.79 & 0.07 \\
\hline Post-hypnosis anticipation 2 & 0.77 & 0.09 & 0.82 & 0.09 \\
\hline Post-hypnosis pain task 2 & 0.78 & 0.08 & 0.79 & 0.07 \\
\hline
\end{tabular}

Note: $*_{p}<0.05 ;{ }^{*} p<0.01$. Units for blood flow are normalized and thus arbitrary units. Abbreviation: SCD, sickle-cell disease. 
post-hypnosis pain threshold (median 46.3) was higher than pre-hypnosis threshold (median 44.1) in the control group $(Z=-2.542, r=0.68 ; p=0.011)$, but pre- and post-hypnosis pain threshold (median 43.8 and 45.5 , respectively) did not differ in the SCD group $(Z=-0.722, r=0.21 ; p=0.43)$. Similarly, results revealed that pain tolerance significantly increased following hypnosis in the control group $\left(t_{13}=2.49, d=0.67 ; p=0.027\right)$, but not in the SCD group $(Z=-0.0408, r=0.097 ; p=0.72)$. Finally, post-hypnosis pain-intensity ratings (median 18.75) were lower than pre-hypnosis ratings (median 26.25) in the control group $(Z=-2.275, r=-0.55 ; p=0.041)$. Post-hypnosis pain-intensity ratings decreased in the SCD group, as indicated by a moderate effect size, but did not reach statistical significance $\left(t_{13}=-1.825, d=-0.49 ; p=0.091\right)$.

\section{Aim 3: effects of hypnosis on peripheral blood-flow responsiveness}

A paired-sample $t$-test revealed that in the SCD group, anticipation-period peripheral blood-flow amplitude was significantly higher following hypnosis $\left(t_{10}=5.722, d=1.73\right.$; $p=0.0002)$. In the control group, there was no change between pre- and post-hypnosis anticipation-period peripheral blood flow $\left(t_{12}=-0.0207, d=0.06, p=0.84\right)$. There were no other pre- and post-hypnosis differences in each task for either group. See Table 2.

\section{Aim 4: effect of hypnosis across groups}

An independent-sample $t$-test across groups revealed that the increases in blood-flow amplitude during post-hypnosis pain task 1 - anticipation, pain task 1 (trending toward significance, moderate effect size), and pain task 2 - anticipation period (trending toward significance, moderate effect size) were larger in the SCD group compared to controls $(t=3.152$, $d=1.242, p=0.004 ; t=1.704, d=0.68, p=0.1 ; t=1.403, d=0.56$, $p=0.18)$. Change in blood-flow amplitude during the painintensity task was similar across groups (Table 3 ).

\section{Discussion}

The current pilot study assessed the effects of a brief hypnosis session on acute experimental pain responses and peripheral blood flow in adults with and without SCD. Overall, following hypnosis, pain threshold and tolerance increased and pain intensity decreased in the control group, and in the SCD group peripheral blood flow increased to levels comparable to controls. Pain-threshold and tolerance levels did not change following hypnosis in patients with SCD, but a moderate effect size showed decreased pain intensity in this group.

\section{Acute-pain responsiveness}

Examination of baseline pain responsiveness revealed that there were no differences between controls and adults with $\mathrm{SCD}$ in respect to pain threshold, tolerance, or intensity. We found that following hypnosis, pain threshold and tolerance significantly increased and pain intensity decreased in the control group. Pain-threshold and tolerance levels did not change following hypnosis in patients with SCD. There was a trend toward decreased pain intensity in the SCD group, and although this decrease was not statistically significant, there was a moderate effect in the hypothesized direction. Of note, based on previously published clinically significant benchmarks for change in pain intensity, the mean decrease in pain intensity in the control group reflected a minimally

Table 2 Effects of hypnosis flow on peripheral blood flow responsiveness

\begin{tabular}{|c|c|c|c|c|c|c|}
\hline & \multicolumn{3}{|c|}{ Control } & \multicolumn{3}{|l|}{ SCD } \\
\hline & $\boldsymbol{t}$ & $p$ & d & $t$ & $p$ & d \\
\hline Pre-hypnosis anticipation period & -0.207 & 0.84 & 0.06 & 5.722 & 0.0002 & 1.73 \\
\hline Pre-hypnosis pain task & -0.01 & 0.99 & 0.003 & -0.587 & 0.57 & 0.18 \\
\hline Post-hypnosis anticipation period & 0.187 & 0.85 & 0.05 & 1.294 & 0.23 & 0.39 \\
\hline Post-hypnosis pain task & 0.255 & 0.8 & 0.07 & 0.719 & 0.49 & 0.22 \\
\hline
\end{tabular}

Abbreviation: SCD, sickle-cell disease.

Table 3 Effect of hypnosis on peripheral blood flow

\begin{tabular}{|c|c|c|c|c|c|c|c|}
\hline \multicolumn{8}{|l|}{ Change in blood-flow amplitude } \\
\hline \multirow[t]{2}{*}{ Post-hypnosis period } & \multicolumn{2}{|c|}{ Control $(n=13)$} & \multicolumn{2}{|c|}{$\operatorname{SCD}(n=I I)$} & \multirow[t]{2}{*}{$t$} & \multirow[t]{2}{*}{$p$} & \multirow[t]{2}{*}{$d$} \\
\hline & Mean & SD & Mean & SD & & & \\
\hline Pre-hypnosis anticipation period & -0.01 & 0.068 & 0.06 & 0.042 & -3.152 & 0.004 & 1.242 \\
\hline Pre-hypnosis pain task & -0.01 & 0.049 & 0.02 & 0.035 & -1.704 & 0.103 & 0.679 \\
\hline Post-hypnosis anticipation period & -0.01 & 0.088 & 0.03 & 0.067 & -1.403 & 0.175 & 0.561 \\
\hline Post-hypnosis pain task & -0.01 & 0.026 & 0 & 0.076 & -0.251 & 0.806 & 0.11 \\
\hline
\end{tabular}

Abbreviation: SCD, sickle-cell disease. 
important change, ${ }^{23}$ whereas the SCD group did not reach this threshold. ${ }^{24}$ This pilot study may have had insufficient power to detect a significant effect, and there was limited variability in pain scores across each group. Future work may benefit from including a larger sample size to examine the clinically significant effects of hypnosis on pain outcomes.

Another explanation for the lack of reduction in pain thresholds and tolerance in the SCD group may be the presence of central sensitization resulting in persistent or chronic pain. Persistent pain often experienced by individuals with $\mathrm{SCD}^{2}$ may result in altered pain perception set points. Modulation of neuronal activation thresholds have also been observed via TRPV1 channels, which may translate to changes in pain sensitivity to thermal stimuli ${ }^{21}$ and changes in the central gain of the somatosensory system. ${ }^{22}$ However, if patients with SCD are exposed to background chronic pain, they may use a different reference point to indicate acute-pain responses than those who do not have this experience with chronic pain. Pain thresholds may be set higher in patients with SCD compared to controls, because of either past experiences with significant pain during VOCs or because of underlying chronic pain and altered pain perception. Therefore, the limited dose of treatment (ie, a single, brief hypnosis session) may have not been sufficient to alter acute-pain thresholds and tolerance in the face of altered peripheral and central pain-modulation systems secondary to pain history or the development of chronic pain. Future studies would benefit from assessing pain history and the presence of chronic pain to determine how these may affect acute-pain responsiveness. Mechanisms of acute pain and chronic pain in patients with SCD warrant further study.

\section{Peripheral blood flow}

Although pain responses in the SCD group did differ significantly following hypnosis, the single hypnosis session did have a significant effect on peripheral blood flow in anticipation of and in response to pain stimulation in the SCD group. At baseline, patients with SCD had significantly lower blood flow than controls, but these levels increased to levels comparable to the control group during post-hypnosis tasks, erasing the group differences found at baseline. A change in blood flow with hypnosis was not seen in the control group. Hypnosis may produce analgesia by engaging descending inhibitory pathways from the brain and increasing regional blood flow. ${ }^{23}$ In the current study, we expected blood flow in both groups to increase with hypnosis, but blood flow increased only in the SCD group. In this study, we demonstrated that hypnosis was beneficial in improving peripheral blood flow in anticipation of pain stimulation in patients with SCD.

The current findings provide further support that patients with SCD exhibit peripheral vascular system dysfunction. ${ }^{24}$ Our results indicate that a single brief hypnosis session may activate top-down neuromodulatory mechanisms in patients with SCD that result in increased vasodilation and peripheral blood flow to a degree comparable to that of healthy controls. Longitudinal research utilizing multiple hypnosis sessions and continuous monitoring of blood flow in a larger sample is warranted to examine the efficacy of hypnosis on blood flow over time. Of interest is that peripheral blood flow increased with hypnosis in anticipation of the pain event in SCD patients, even without changes in reported pain responses. Exploration of effects of hypnosis on pathways, such as the autonomic nervous system (ANS), that can impact peripheral blood flow but may not reach conscious awareness in patients with SCD warrant further study.

\section{Possible mechanisms}

Considering possible mechanisms from a central perspective, alterations in brain connectivity in inhibitory pain-control networks have been observed during hypnosis. ${ }^{25,26}$ Specifically, increased connectivity between the ipsilateral insula and bilateral dorsolateral prefrontal cortex has been observed during hypnosis in healthy individuals who were highly hypnotizable. ${ }^{25}$ In addition, decreased fractional amplitude of low-frequency fluctuation in the dorsal anterior cingulate cortex was observed in individuals who were highly hypnotizable, a finding that may represent decreased attention to the external environment during hypnosis ${ }^{25}$ and thus decreased pain perception and responsiveness. Results from these studies provide more evidence to support the concept that hypnosis affects pain-modulatory systems, engages top-down neuromodulatory pain circuits, and helps filter out external stressors that may be contributing to allostatic stress load (ie, pain and anticipation of pain). In the current study, although no significant changes were observed in behavioral responses, the increase in blood flow despite exposure to stress (ie, a pain stimulus) may demonstrate this central pain-modulatory mechanism.

In our sample, patients with SCD had lower baseline peripheral blood flow compared to healthy controls, which is consistent with other literature showing evidence of allostatic load and ANS dysfunction in patients with SCD. ${ }^{27}$ In the current study, following a single session of hypnosis, patients with SCD exhibited increased peripheral blood flow in anticipation of and during pain tasks. This finding suggests that a 
single session of hypnosis may affect peripheral vascular pain responses modulated by the ANS. Given that ANS-modulated peripheral vasoconstriction with stress or pain may increase likelihood for vaso-occlusion and VOCs, ${ }^{28-32}$ further examination of ANS mechanisms and neuromodulatory treatments aimed at addressing pain-related autonomic regulation (eg, hypnosis) is warranted.

Additional research is needed to understand the underlying mechanisms of the effects of hypnosis in patients with SCD. Specifically, future studies should examine neurally mediated systems, such as the ANS (eg, heart-rate variability and electrodermal activity), and central supraspinal parameters (eg, brain imaging) to advance our understanding of treatment effects and SCD pain-related pathophysiology from a multisystem perspective. Tailoring neuromodulatory interventions for the specific needs of patients with SCD has the potential to improve treatment and patient outcomes.

\section{Limitations}

Limitations in the study should be noted. First, the small sample of this pilot study limited statistical power, and findings may not generalize to larger samples or broader populations. In addition, this pilot study did not include a treatment-control condition, which limits our ability to assess the success or unique effects of hypnosis. Future work should include a treatment-control condition (eg, diaphragmatic breathing, distraction) within a randomized controlled trial to examine and confirm the specific effects of hypnosis. Future studies may also compare hypnosis to other evidence-based interventions, such as biofeedback, cognitive-behavioral therapy, or therapeutic yoga. ${ }^{33}$ The current study did not examine the effects of hypnosis on clinical pain outcomes either, including the frequency and/or severity of VOCs, and future work would benefit from assessing these outcomes through longitudinal methodology. Further, it is possible that more than a single hypnosis session is needed to affect change in perception and behavioral responses to acute pain, and the examination of the effects of multiple hypnosis sessions on pain outcomes is warranted.

In the current study, baseline peripheral blood flow was related to age in patients with SCD. This finding is not surprising, since we would expect that as individuals age they are more prone to microvascular pathologies, ${ }^{34}$ which may impair vascular function. Therefore, potential previous vascular damage in our adult sample may have influenced baseline data and treatment effects. Sampling of adolescents within a specific age range in future studies may help control for potential effects of microvascular damage on blood-flow outcomes.
It is also important to note that only thermal sensory testing was applied in the current study, and may not be generalizable to all types of pain, as thermal pain has different underlying molecular mechanisms compared to other types of pain. ${ }^{35}$ Additional covariates that may influence ANS responsiveness and blood flow not included in the current analyses (eg, pain history, SCD genotype, history of VOCs, heart disease, anxiety, depression, smoking history, and medication use) may have had an impact on the current findings and should be assessed in future work. In addition, the current study did not assess hypnotizability, which has been shown to be associated with the effect of hypnosis on autonomic response ${ }^{10}$ and vaso-occlusion. ${ }^{36}$ The inclusion of qualitative post-hypnosis interviews in future work may help further explore participants' experiences and the effect of hypnosis. Additionally, investigating the anticipation period to unpainful stimuli would help address differences and similarities in physiological responses. To help diminish the effect of confounding variables, counterbalancing hypnotic and nonhypnotic conditions may also help better isolate the effect of hypnosis. Finally, the investigator and clinician in the current study were not blinded to the patient condition, which may have introduced bias. Future work will aim to blind the investigator in the room or place the investigator in a different room to collect psychophysiological measures.

\section{Conclusion}

This study demonstrated that the amount of peripheral blood flow in anticipation of pain in adults with SCD increased following a single, 30-minute hypnosis session. There was a trend toward decreased perceived pain in SCD patients as well. Given that peripheral vasoconstriction and blood flow likely play a role in the development of VOCs, these findings provide initial support for further study of mechanisms and effects of neuromodulatory interventions in pain management for patients with SCD. Collectively, our results suggest that patients with SCD may need targeted treatment that addresses both central and peripheral neurovascular processes. Future work will determine if engagement in hypnotherapy affects long-term pain and VOC outcomes in patients with SCD, as well as examine pathways through which these effects take place.

\section{Author contributions}

RRB was the primary author of manuscript and conducted statistical analyses and interpretation of data. SRM was the secondary author of the manuscript, interpreted data, and provided expertise regarding sickle-cell disease. SE was 
the lead developer of the hypnosis protocol and conducted the hypnosis procedure with participants. KL assisted with development of laboratory assessment and conducted laboratory assessments with participants. TDC provided expertise regarding clinical aspects of sickle-cell disease. LKZ assisted with development of the overall study protocol and provided clinical expertise regarding the use of hypnosis for pain. JCT provided expertise on laboratory pain assessment and was the lead developer of the laboratory pain protocol. All authors contributed toward data analysis, drafting and revising the paper and agree to be accountable for all aspects of the work.

\section{Acknowledgment}

This research was supported by the National Heart, Lung, and Blood Institute (1U54HL117718 to TDC and LKZ).

\section{Disclosure}

The abstract of this paper was presented at the 2016 American Society of Hematology meeting as a poster with interim findings. The authors report no conflicts of interest in this work.

\section{References}

1. Lanzkron S, Carroll CP, Haywood C Jr. Mortality rates and age at death from sickle cell disease: U.S., 1979-2005. Public Health Rep. 2013;128:110-116.

2. Borsook D, Maleki N, Becerra L, McEwen B. Understanding migraine through the lens of maladaptive stress responses: a model disease of allostatic load. Neuron. 2012;73:219-234.

3. Ballas SK, Gupta K, Adams-Graves P. Sickle cell pain: a critical reappraisal. Blood. 2012;120:3647-3656.

4. Brandow AM, Stucky CL, Hillery CA, Hoffmann RG, Panepinto JA. Patients with sickle cell disease have increased sensitivity to cold and heat. Am J Hematol. 2013;88:37-43.

5. Kohli DR, Li Y, Khasabov SG, et al. Pain-related behaviors and neurochemical alterations in mice expressing sickle hemoglobin: modulation by cannabinoids. Blood. 2010;116:456-465.

6. Weyer AD, Zappia KJ, Garrison SR, O'Hara CL, Dodge AK, Stucky CL. Nociceptor sensitization depends on age and pain chronicity. eNeuro. 2016;3: 0115 .

7. Zeltzer L, Lebaron S. Hypnosis and non hypnotic techniques for reduction of pain and anxiety during painful procedures in children and adolescents with cancer. $J$ Pediatr. 1982;101:1032-1035.

8. Jensen MP. The neurophysiology of pain perception and hypnotic analgesia: implications for clinical practice implications for clinical practice. Am J Clin Hypn. 2008;51:123-148.

9. Jensen MP, Adachi T, Tomé-Pires C, Lee J, Jamil Osman Z, Miró J. Mechanisms of hypnosis: toward the development of a biopsychosocial model. Int J Clin Exp Hypn. 2015;63:34-75.

10. DeBenedittis G, Cigada M, Bianchi A, Signorini MG, Cerutti S. Autonomic changes during hypnosis: a heart rate variability power spectrum analysis as a marker of sympatho-vagal balance. Int J Clin Exp Hypn. 1994;42:140-152.

11. Palsson OS, Turner MJ, Johnson DA, Burnett CK, Whitehead WE. Hypnosis treatment for severe irritable bowel syndrome: investigation of mechanism and effects on symptoms. Dig Dis Sci. 2002;47:2605-2614.
12. Dinges DF, Whitehouse WG, Orne EC, et al. Self-hypnosis training as an adjunctive treatment in the management of pain associated with sickle cell disease. Int J Clin Exp Hypn. 1997;45:417-432.

13. Zeltzer L, Dash J, Holland JP. Hypnotically induced pain control in sickle cell anemia. Pediatrics. 1979;64:533-536.

14. Casiglia E, Rossi A, Tikhonoff V, et al. Local and systemic vasodilation following hypnotic suggestion of warm tub bathing. Int J Psychophysiol. 2006;62:60-65.

15. Moore LE, Wiesner SL. Hypnotically-induced vasodilation in the treatment of repetitive strain injuries. Am J Clin Hypn. 1996;39:97-104.

16. Grabowska M. The effect of hypnosis and hypnotic suggestion on the blood flow in the extremities. Pol Med J. 1971;10:1044-1051.

17. Mittleman K, Doubt T, Gravitz M. Influence of self-induced hypnosis on thermal responses during immersion in 25 degrees $\mathrm{C}$ water. Aviat Sp Env Med. 1992;63:689-695.

18. Porro CA, Baraldi P, Pagnoni G, et al. Does anticipation of pain affect cortical nociceptive systems? J Neurosci. 2002;22:3206-3214.

19. Khaleel M, Puliyel M, Sunwoo J, et al. Thermal pain and pain anticipation induce a decrease in microvascular perfusion in sickle cell and normal subjects. Blood. 2015;126:67.

20. Jensen M. Hypnosis for Chronic Pain Management: Workbook. New York: Oxford University Press; 2011.

21. Cohen J. Statistical Power Analysis for the Behavioral Sciences. New Jersey: Lawrence Erlbaum; 1977.

22. Cohen J. A power primer. Psychol Bull. 1992;112:155-159.

23. Dworkin RH, Turk DC, Wyrwich KW, et al. Interpreting the clinical importance of treatment outcomes in chronic pain clinical trials: IMMPACT recommendations. J Pain. 2008;9:105-121.

24. Farrar JT, Young JP, Lamoreaux L, Werth JL, Poole RM. Clinical importance of changes in chronic pain intensity measured on an 11-point numerical pain rating scale. Pain. 2001;94:149-158.

25. Smith WR, Penberthy LT, Bovbjerg VE, et al. Daily assessment of pain in adults with sickle cell disease. Ann Intern Med. 2008;148:94-101.

26. Lutz B, Meiler SE, Bekker A, Tao YX. Updated mechanisms of sickle cell disease-associated chronic pain. Transl Perioper Pain Med. 2015;2: $8-17$.

27. Woolf CJ. Central sensitization: implications for the diagnosis and treatment of pain. Pain. 2011;152:S2-S15.

28. Crawford HJ, Gur RC, Skolnick B, Gur RE, Benson DM. Effects of hypnosis on regional cerebral blood flow during ischemic pain with and without suggested hypnotic analgesia. Int J Psychophysiol. 1993;15: 181-195.

29. Sangkatumvong S, Khoo MC, Kato R, et al. Peripheral vasoconstriction and abnormal parasympathetic response to sighs and transient hypoxia in sickle cell disease. Am J Respir Crit Care Med. 2011;184: 474-481.

30. Jiang H, White MP, Greicius MD, Waelde LC, Spiegel D. Brain activity and functional connectivity associated with hypnosis. Cereb Cortex. Epub 2016 July 28.

31. Vanhaudenhuyse A, Laureys S, Faymonville ME. Neurophysiology of hypnosis. Neurophysiol Clin. 2014;44:343-353.

32. Connes P, Coates TD. Autonomic nervous system dysfunction: implication in sickle cell disease. C R Biol. 2013;336:142-147.

33. Zelzter L, Zeltzer P. Pain in Children and Young Adults: The Journey Back to Normal. Encino (CA): Shilysca Press; 2016.

34. Brown WR, Thore CR. Review: cerebral microvascular pathology in aging and neurodegeneration. Neuropathol Appl Neurobiol. 2011;37: 56-74.

35. Julius D, Basbaum AI. Molecular mechanisms of nociception. Nature. 2001;413:203-210.

36. Jambrik Z, Santarcangelo EL, Rudisch T, Varga A, Forster T, Carli G. Modulation of pain-induced endothelial dysfunction by hypnotisability. Pain. 2005;116:181-186. 
The Journal of Pain Research is an international, peer reviewed, open access, online journal that welcomes laboratory and clinical findings in the fields of pain research and the prevention and management of pain. Original research, reviews, symposium reports, hypothesis formation and commentaries are all considered for publication.
The manuscript management system is completely online and includes a very quick and fair peer-review system, which is all easy to use. Visit http://www.dovepress.com/testimonials.php to read real quotes from published authors. 